Supporting Information for

\title{
Compound-specific stable carbon isotope ratios of terrestrial biomarkers in urban aerosols from Beijing, China
}

\author{
Lujie Ren ${ }^{1,2}$, Wei Hul, Juzhi Hou', Linjie Li ${ }^{2,4}$, Siyao Yue ${ }^{2,4}$, Yele
}

Sun ${ }^{2}$, Zifa Wang ${ }^{2}$, Xiaodong Li $^{1}$, Chandra Mouli Pavuluri ${ }^{1}$, Shengjie Hou²,

Cong-Qiang Liu ${ }^{1}$, Kimitaka Kawamura ${ }^{5}$, Robert Mark Ellam ${ }^{6}$, and

$$
\text { Pingqing } \mathrm{Fu}^{1, *}
$$

${ }^{1}$ Institute of Surface-Earth System Science, Tianjin University, Tianjin 300072, China

${ }^{2}$ LAPC, Institute of Atmospheric Physics, Chinese Academy of Sciences, Beijing 100029, China

${ }^{3}$ Institute of Tibetan Plateau Research, Chinese Academy of Sciences, Beijing 100085, China

${ }^{4}$ College of Earth Sciences, University of Chinese Academy of Sciences, Beijing 100049, China

${ }^{5}$ Chubu Institute for Advanced Studies, Chubu University, Kasugai, Japan

${ }^{6}$ Scottish Universities Environmental Research Centre, Glasgow, Scotland, UK

${ }^{7}$ Center for Excellence in Regional Atmospheric Environment, Institute of Urban Environment, Chinese Academy of Sciences, Xiamen 361021, China

*Corresponding author e-mail: fupingqing@tju.edu.cn

This supporting information contains 14 pages with 4 Tables, 6 Figures and references. 
Table S1. Signal-to-noise ratios $(\mathrm{S} / \mathrm{N})$, limits of detection (LOD) and relative standard deviations (RSD) based on duplicate analysis $(n=4)$.

\begin{tabular}{|c|c|c|c|c|c|c|c|}
\hline$n$-Alkanes & $\mathrm{S} / \mathrm{N}$ & LOD (ppm) & RSD $(\%)$ of $\mathrm{S} / \mathrm{N}$ & Fatty acids & $\mathrm{S} / \mathrm{N}$ & LOD (ppm) & $\operatorname{RSD}(\%)$ of $\mathrm{S} / \mathrm{N}$ \\
\hline $\mathrm{C}_{13}$ & 594 & 0.101 & 11.3 & $\mathrm{C}_{10: 0}$ & 221 & 0.007 & 13.4 \\
\hline $\mathrm{C}_{19}$ & 835 & 0.072 & 9.67 & $\mathrm{C}_{11: 0}$ & 536 & 0.004 & 14.9 \\
\hline $\mathrm{C}_{20}$ & 1233 & 0.049 & 5.15 & $\mathrm{C}_{12: 0}$ & 209 & 0.012 & 14.2 \\
\hline $\mathrm{C}_{21}$ & 464 & 0.129 & 10.92 & $\mathrm{C}_{13: 0}$ & 102 & 0.222 & 10.6 \\
\hline $\mathrm{C}_{22}$ & 531 & 0.113 & 8.62 & $\mathrm{C}_{14: 0}$ & 234 & 0.067 & 12.7 \\
\hline $\mathrm{C}_{23}$ & 1065 & 0.056 & 5.21 & $\mathrm{C}_{15: 0}$ & 236 & 0.018 & 12.6 \\
\hline $\mathrm{C}_{24}$ & 2061 & 0.029 & 3.49 & $\mathrm{C}_{16: 0}$ & 2648 & 0.148 & 5.57 \\
\hline $\mathrm{C}_{25}$ & 3453 & 0.017 & 4.64 & $\mathrm{C}_{17: 0}$ & 88 & 0.101 & 12.5 \\
\hline $\mathrm{C}_{26}$ & 2628 & 0.023 & 4.22 & $\mathrm{C}_{18: 0}$ & 280 & 0.224 & 5.35 \\
\hline $\mathrm{C}_{27}$ & 2308 & 0.026 & 3.51 & $\mathrm{C}_{19: 0}$ & 31 & 0.093 & 11.2 \\
\hline $\mathrm{C}_{28}$ & 1106 & 0.054 & 7.31 & $\mathrm{C}_{20: 0}$ & 889 & 0.022 & 9.79 \\
\hline $\mathrm{C}_{29}$ & 712 & 0.084 & 10.5 & $\mathrm{C}_{21: 0}$ & 153 & 0.044 & 15.6 \\
\hline $\mathrm{C}_{30}$ & 2554 & 0.023 & 5.45 & $\mathrm{C}_{22: 0}$ & 657 & 0.053 & 13.4 \\
\hline $\mathrm{C}_{31}$ & 2635 & 0.023 & 3.64 & $\mathrm{C}_{23: 0}$ & 172 & 0.114 & 17.2 \\
\hline $\mathrm{C}_{32}$ & 2262 & 0.027 & 4.26 & $\mathrm{C}_{24: 0}$ & 398 & 0.137 & 7.5 \\
\hline $\mathrm{C}_{33}$ & 1741 & 0.034 & 5.36 & $\mathrm{C}_{25: 0}$ & 221 & 0.065 & 13.4 \\
\hline $\mathrm{C}_{34}$ & 1115 & 0.054 & 7.24 & $\mathrm{C}_{26: 0}$ & 195 & 0.219 & 15.2 \\
\hline $\mathrm{C}_{35}$ & 959 & 0.063 & 8.42 & $\mathrm{C}_{27: 0}$ & 38 & 0.254 & 15.4 \\
\hline $\mathrm{C}_{36}$ & 540 & 0.111 & 12.1 & $\mathrm{C}_{28: 0}$ & 188 & 0.262 & 15.7 \\
\hline $\mathrm{C}_{37}$ & 436 & 0.138 & 13.9 & $\mathrm{C}_{29: 0}$ & 79 & 0.115 & 14.2 \\
\hline - & - & - & - & $\mathrm{C}_{30: 0}$ & 220 & 0.204 & 13.5 \\
\hline- & - & - & - & $\mathrm{C}_{31: 0}$ & 85 & 0.035 & 11.2 \\
\hline - & - & - & - & $\mathrm{C}_{32: 0}$ & 97 & 0.169 & 11.9 \\
\hline
\end{tabular}


Table S2. Concentrations and other parameters of $n$-alkanes and fatty acids in aerosol samples (TSP) collected in Beijing.

\begin{tabular}{|c|c|c|c|c|c|c|c|c|c|c|}
\hline & \multicolumn{2}{|c|}{ Whole period } & \multicolumn{2}{|c|}{ Spring } & \multicolumn{2}{|c|}{ Summer } & \multicolumn{2}{|c|}{ Fall } & \multicolumn{2}{|c|}{ Winter } \\
\hline & Range & Average \pm SD & Range & Average \pm SD & Range & Average \pm SD & Range & Average \pm SD & Range & Average \pm SD \\
\hline \multicolumn{11}{|c|}{ n-Alkanes } \\
\hline Conc.(ng m ${ }^{-3}$ ) & $34-360$ & $152 \pm 91.4$ & $86.3-264$ & $155 \pm 50.3$ & $34.0-89.4$ & $57.8 \pm 19$ & $75.1-162$ & $114 \pm 27.4$ & $173-360$ & $281 \pm 51.3$ \\
\hline $\mathrm{CPI}^{\mathrm{a}}$ & $1.15-6.07$ & $2.37 \pm 1.25$ & $1.85-6.07$ & $3.99 \pm 1.32$ & $1.42-3.1$ & $2.03 \pm 0.53$ & $1.66-2.99$ & $2.26 \pm 0.46$ & $1.15-1.38$ & $1.21 \pm 0.07$ \\
\hline $\mathrm{CPI}_{\mathrm{LMW}}{ }^{\mathrm{b}}$ & $0.71-1.44$ & $0.93 \pm 0.2$ & $0.71-1.44$ & $1.02 \pm 0.27$ & $0.4-1.49$ & $0.88 \pm 0.29$ & $0.79-1.03$ & $0.92 \pm 0.08$ & $0.86-0.9$ & $0.89-0.03$ \\
\hline $\mathrm{CPI}_{\mathrm{HMW}}{ }^{\mathrm{c}}$ & $1.92-9$ & $3.49 \pm 1.83$ & $2.45-9$ & $5.8 \pm 2.13$ & $1.92-3.02$ & $2.75 \pm 0.9$ & $2.25-4.12$ & $3.24 \pm 0.75$ & $2.15-0.2$ & $1.84-2.42$ \\
\hline WNA\% & $8.67-74.8$ & $35 \pm 19.3$ & $31.6-74.8$ & $57.9 \pm 12.5$ & $17.2-51.2$ & $33.3 \pm 11.4$ & $26.3-50.9$ & $37.9 \pm 8.67$ & $8.67-16.1$ & $10.7 \pm 2.21$ \\
\hline HMW/LMW & $0.63-18.1$ & $6.95 \pm 4.87$ & $7.18-18.1$ & $11.6 \pm 3.50$ & $2.77-17.3$ & $8.97 \pm 3.79$ & $3.36-9.73$ & $6.56 \pm 2.03$ & $0.63-0.97$ & $0.71 \pm 0.10$ \\
\hline \multicolumn{11}{|c|}{ Fatty acids } \\
\hline Conc.(ng m $\left.{ }^{-3}\right)$ & $158-934$ & $382 \pm 168$ & $158-494$ & $356 \pm 110$ & $178-329$ & $263 \pm 54$ & 359-934 & $553 \pm 217$ & $206-556$ & $356 \pm 102$ \\
\hline CPI & $3.06-7.50$ & $4.75 \pm 1.05$ & $4.26-7.5$ & $5.46 \pm 1.12$ & $4.83-7.1$ & $5.56 \pm 0.62$ & $3.41-4.87$ & $4.3 \pm 0.48$ & $3.06-4.50$ & $3.67 \pm 0.37$ \\
\hline LMW/HMW & $1.15-12.7$ & $4.24 \pm 2.67$ & $1.15-3.17$ & $2.33 \pm 0.70$ & $3.49-12.7$ & $7.42 \pm 3.12$ & $1.58-6.91$ & $4.07 \pm 1.81$ & $2.50-4.70$ & $3.16 \pm 0.88$ \\
\hline \multicolumn{11}{|c|}{ Organic carbon } \\
\hline
\end{tabular}




\begin{tabular}{lccccccccccccc}
\hline OC $\left(\mu \mathrm{g} \mathrm{m}^{-3}\right)$ & $7.2-40.2$ & $21.8 \pm 9.82$ & $16-30.9$ & $23.1 \pm 4.68$ & $7.2-12.4$ & $9.17 \pm 1.84$ & $13.1-38.8$ & $24.3 \pm 8.35$ & $20.5-40.2$ & $30.4 \pm 7.37$ \\
$n$-Alkanes/OC (\%) & $0.21-1.35$ & $0.59 \pm 0.24$ & $0.35-0.89$ & $0.57 \pm 0.14$ & $0.21-0.87$ & $0.51 \pm 0.19$ & $0.22-0.64$ & $0.42 \pm 0.13$ & $0.61-1.35$ & $0.87 \pm 0.22$ \\
Fatty acids/OC (\%) & $0.51-2.69$ & $1.41 \pm 0.64$ & $0.51-1.64$ & $1.11 \pm 0.33$ & $0.61-3.19$ & $1.97 \pm 0.67$ & $1.01-2.69$ & $1.59 \pm 0.49$ & $0.54-2.18$ & $0.94 \pm 0.47$ \\
\hline
\end{tabular}

${ }^{\text {a }} \mathrm{CPI}$, carbon preference index, $\Sigma$ odd $\left(\mathrm{C}_{21-35}\right) / \Sigma$ even $\left(\mathrm{C}_{20-36}\right)$ for $n$-alkanes; $\Sigma$ even $\left(\mathrm{C}_{20-32}\right) / \Sigma$ odd $\left(\mathrm{C}_{21-31}\right)$ for fatty acids.

${ }^{\mathrm{b}}$ WNA\%: wax $n$-alkanes percentage, calculated as $\left[\Sigma \mathrm{C}_{n}-0.5\left(\mathrm{C}_{\mathrm{n}-1}+\mathrm{C}_{\mathrm{n}+1}\right)\right] / \Sigma n$-alkanes. 
Table S3. Stable carbon isotopic compositions $\left(\delta^{13} \mathrm{C}\right)$ of individual $n$-alkanes and fatty acids.

\begin{tabular}{|c|c|c|c|c|c|c|c|c|c|c|c|c|}
\hline & \multicolumn{3}{|c|}{ Spring } & \multicolumn{3}{|c|}{ Summer } & \multicolumn{3}{|c|}{ Fall } & \multicolumn{3}{|c|}{ Winter } \\
\hline & Min & Max & Average & Min & Max & Average & Min & Max & Average & Min & Max & Average \\
\hline \multicolumn{13}{|c|}{ n-Alkanes (\%o vs. VPDB) } \\
\hline $\mathrm{C}_{23}$ & -33.6 & -30.5 & -32.0 & -32.9 & -28.8 & -31.0 & -36.9 & -29.4 & -33.3 & -27.6 & -26.1 & -27.1 \\
\hline $\mathrm{C}_{24}$ & -32.3 & -29.0 & -30.5 & -34.1 & -27.8 & -30.4 & -35.1 & -29.0 & -31.2 & -27.7 & -25.9 & -26.9 \\
\hline $\mathrm{C}_{25}$ & -33.8 & -30.7 & -32.1 & -34.8 & -28.1 & -31.3 & -35.9 & -30.0 & -32.9 & -30.6 & -26.7 & -28.6 \\
\hline $\mathrm{C}_{26}$ & -32.4 & -29.5 & -30.8 & -33.8 & -28.0 & -30.4 & -33.9 & -30.6 & -32.3 & -29.1 & -26.9 & -28.4 \\
\hline $\mathrm{C}_{27}$ & -33.4 & -30.9 & -31.9 & -35.4 & -28.7 & -31.9 & -35.0 & -31.3 & -33.5 & -30.9 & -28.3 & -29.7 \\
\hline $\mathrm{C}_{28}$ & -34.0 & -29.9 & -31.9 & -40.6 & -27.8 & -33.4 & -36.1 & -31.5 & -33.6 & -32.5 & -26.4 & -28.2 \\
\hline $\mathrm{C}_{29}$ & -33.8 & -31.8 & -32.9 & -36.8 & -30 & -33.0 & -35.7 & -32.3 & -34.3 & -37.3 & -30.9 & -34.0 \\
\hline $\mathrm{C}_{30}$ & -33.6 & -29.8 & -31.7 & -33.7 & -27.3 & -31.0 & -35.1 & -31.3 & -33.3 & -43.1 & -28.3 & -32.7 \\
\hline $\mathrm{C}_{31}$ & -34.8 & -32.4 & -33.8 & -37.7 & -29.4 & -34.1 & -36.8 & -32.4 & -34.6 & -36.3 & -31.4 & -33.3 \\
\hline $\mathrm{C}_{32}$ & -36.2 & -30.9 & -33.6 & -36.5 & -28.2 & -33.2 & -38.9 & -32.5 & -35.3 & -26.5 & -25.2 & -25.8 \\
\hline $\mathrm{C}_{33}$ & -34.9 & -32.4 & -33.9 & -36.2 & -28.8 & -33.4 & -36.7 & -32.2 & -34.9 & -35.5 & -30.1 & -33.3 \\
\hline $\mathrm{C}_{34}$ & -33.4 & -31.3 & -32.4 & -40.1 & -29.4 & -33.8 & -37.9 & -31.4 & -34.3 & nd & nd & nd \\
\hline
\end{tabular}




\begin{tabular}{|c|c|c|c|c|c|c|c|c|c|c|c|c|}
\hline \multicolumn{13}{|c|}{ Fatty acids (\%o vs. VPDB) } \\
\hline $\mathrm{C}_{14: 0}$ & -29.9 & -26.3 & -27.8 & -28.2 & -25.4 & -26.5 & -26.5 & -22.6 & -25.0 & -25.4 & -23.9 & -24.7 \\
\hline $\mathrm{C}_{15: 0}$ & -32.2 & -26.9 & -28.7 & -25.0 & -24.4 & -24.7 & -29.2 & -22.9 & -25.5 & -25.7 & -24.6 & -25.2 \\
\hline $\mathrm{C}_{16: 0}$ & -29.8 & -26.9 & -28.6 & -28.4 & -26.9 & -27.8 & -28.3 & -27.0 & -27.6 & -27.5 & -26.0 & -27.1 \\
\hline $\mathrm{C}_{17: 0}$ & -28.9 & -26.3 & -27.9 & -25.2 & -23.2 & -24.0 & -26.0 & -23.5 & -24.9 & -27.9 & -23.9 & -26.4 \\
\hline $\mathrm{C}_{18: 0}$ & -31.5 & -26.4 & -28.6 & -28.4 & -25.9 & -26.5 & -27.5 & -25.4 & -26.1 & -26.7 & -26.1 & -26.3 \\
\hline $\mathrm{C}_{19: 0}$ & -30.1 & -30.1 & -30.1 & nd & nd & nd & nd & nd & nd & nd & nd & nd \\
\hline $\mathrm{C}_{20: 0}$ & -34.8 & -28.5 & -32.8 & -31.2 & -29.0 & -29.9 & -30.6 & -26.3 & -28.5 & -29.3 & -26.9 & -28.4 \\
\hline $\mathrm{C}_{21: 0}$ & -30.4 & -30.4 & -30.4 & nd & nd & nd & nd & nd & nd & -29.5 & -28.2 & -28.8 \\
\hline $\mathrm{C}_{22: 0}$ & -35.4 & -30.3 & -32.7 & -31.1 & -28.8 & -30.2 & -31.2 & -26.3 & -28.8 & -29.8 & -28.7 & -29.4 \\
\hline $\mathrm{C}_{23: 0}$ & -33.0 & -30.0 & -31.1 & nd & nd & nd & -27.4 & -25.6 & -26.3 & -28.2 & -26.9 & -27.6 \\
\hline $\mathrm{C}_{24: 0}$ & -36.1 & -30.1 & -33.5 & -32.8 & -30.2 & -31.1 & -30.4 & -26.5 & -28.4 & -28.9 & -27.3 & -28.2 \\
\hline $\mathrm{C}_{25: 0}$ & -36.6 & -32.6 & -34.6 & nd & nd & nd & nd & nd & nd & nd & nd & nd \\
\hline $\mathrm{C}_{26: 0}$ & -36.9 & -31.9 & -34.1 & -31.9 & -30.3 & -31.2 & -32.3 & -30.1 & -31.2 & -29.5 & -27.2 & -28.0 \\
\hline $\mathrm{C}_{28: 0}$ & -36.7 & -28.6 & -34.1 & -32.3 & -26.5 & -30.1 & -31.4 & -31.1 & -31.3 & -29.5 & -29.5 & -29.5 \\
\hline
\end{tabular}

nd: not determined 
Table S4. $\delta^{13} \mathrm{C}$ ranges of $n$-alkanes and fatty acids from terrestrial higher plants, ${ }^{1-5}$ combustion of $\mathrm{C}_{4}$ and $\mathrm{C}_{3}$ plants, ${ }^{1,2}$ anthropogenic sources (such as crude oil and vehicle exhaust $)^{6-8}$ and urban aerosols. ${ }^{9-12}$

\begin{tabular}{|c|c|c|c|c|}
\hline Types & Location & CPI & $\delta^{13} \mathrm{C}$ ranges & Main sources \\
\hline \multicolumn{5}{|c|}{$\delta^{13} \mathrm{C}$ ranges of $n$-alkanes } \\
\hline $\mathrm{C}_{3}$ plants & - & $4.3-30.3$ & $-42.1 \%$ to $-30.7 \%$ & - \\
\hline $\mathrm{C}_{4}$ Plants & - & & $-27.4 \%$ to $-17.2 \%$ & - \\
\hline CAM plants & - & & $-34.1 \%$ to $-18 \%$ & - \\
\hline Crude oil & - & - & $-33 \%$ to $-24 \%$ & - \\
\hline Vehicle exhaust & - & - & $-28.7 \%$ to $-27.1 \%$ & - \\
\hline \multirow{4}{*}{ Aerosols } & \multirow{2}{*}{ Chichi-Jima } & \multirow{2}{*}{$1.8-13.1$} & LMW: $-30 \%$ to $-27 \%$ & Fossil fuel \\
\hline & & & HMW: $-34.4 \%$ to $-26.6 \%$ & $\mathrm{C}_{3}$ plants \\
\hline & \multirow{2}{*}{ Tokyo } & \multirow{2}{*}{$1.2-2.1$} & LMW: $-28.6 \%$ to $-25.5 \%$ & Fossil fuel \\
\hline & & & HMW: $-32.8 \%$ to $-27.1 \% 0$ & $\mathrm{C}_{3}$ plants \\
\hline \multirow{8}{*}{ This study } & \multirow{2}{*}{ Spring } & \multirow{2}{*}{$1.85-6.07$} & LMW: $-33.3 \%$ to $-28 \%$ & \\
\hline & & & HMW odd: $-34.8 \%$ to- $30.3 \%$ & \\
\hline & \multirow{2}{*}{ Summer } & \multirow{2}{*}{$1.42-3.1$} & LMW :-34.8\%o to $-27.8 \%$ & \\
\hline & & & $\mathrm{HMW}_{\text {odd: }} \quad-37.6 \%$ to- $28.7 \%$ & \\
\hline & \multirow{2}{*}{ Fall } & \multirow{2}{*}{$1.66-2.99$} & LMW: $-38.1 \%$ to $-29.0 \%$ & \\
\hline & & & $\mathrm{HMW}_{\text {odd: }}-36.7 \%$ to $-31.3 \%$ & \\
\hline & \multirow{3}{*}{ Winter } & \multirow{2}{*}{$1.15-1.38$} & LMW: $-30.5 \%$ to $-25.9 \%$ & \\
\hline & & & HMW odd: $-37.3 \%$ to $-28.3 \%$ o & \\
\hline \multicolumn{4}{|c|}{$\delta^{13} \mathrm{C}$ ranges of fatty acids } & \\
\hline $\mathrm{C}_{3}$ plants & - & - & $-42 \%$ to $-32.4 \%$ o & - \\
\hline $\mathrm{C}_{4}$ Plants & - & - & $-28.2 \%$ to $-19.7 \%$ & - \\
\hline CAM plants & - & - & $-31.7 \%$ to $-21.1 \%$ & - \\
\hline Combustion of $\mathrm{C}_{3}$ plants & - & - & $-38.5 \%$ o to $-26 \%$ & - \\
\hline Combustion of $\mathrm{C}_{4}$ plants & - & - & $-32 \%$ to $-21 \%$ & - \\
\hline \multirow{4}{*}{ Aerosols } & \multirow[t]{2}{*}{ Chichi-Jima } & \multirow{2}{*}{$9.8-24.7$} & LMW: $-27.7 \%$ to $-24 \%$ & \multirow{2}{*}{$\mathrm{C}_{3}$ plants } \\
\hline & & & HMW: $-31.7 \%$ to $-26.5 \%$ & \\
\hline & \multirow[t]{2}{*}{ Hokkaido } & \multirow[t]{2}{*}{-} & LMW: $-25.8 \%$ to $-24.3 \% 0$ & Higher plants and marine organism \\
\hline & & & HMW: $-32 \%$ to $-27.9 \%$ & Higher plants and marine organism \\
\hline \multirow{8}{*}{ This study } & \multirow{2}{*}{ Spring } & \multirow{2}{*}{$4.26-7.5$} & LMW: $-32.2 \%$ o to $-26.2 \%$ 。 & \\
\hline & & & HMW: $-36.9 \%$ o to $-28.5 \%$ o & \\
\hline & \multirow{2}{*}{ Summer } & \multirow{2}{*}{$4.83-7.1$} & LMW: $-28.4 \%$ o to $-23.2 \% \circ$ & \\
\hline & & & HMW: $2-32.8 \%$ o to $-26.5 \%$ 。 & \\
\hline & \multirow{2}{*}{ Fall } & 341487 & LMW: $-29.2 \%$ to $-22.6 \%$ o & 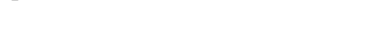 \\
\hline & & $0.41=4.01$ & HMW: $-32.3 \%$ o to $-25.6 \%$ 。 & \\
\hline & Winter & $3.06-4.5$ & LMW: $-28.3 \%$ o to $-23.9 \%$ 。 & \\
\hline & 年 & & HMW: $-29.8 \%$ to $-26.9 \%$ 。 & \\
\hline
\end{tabular}




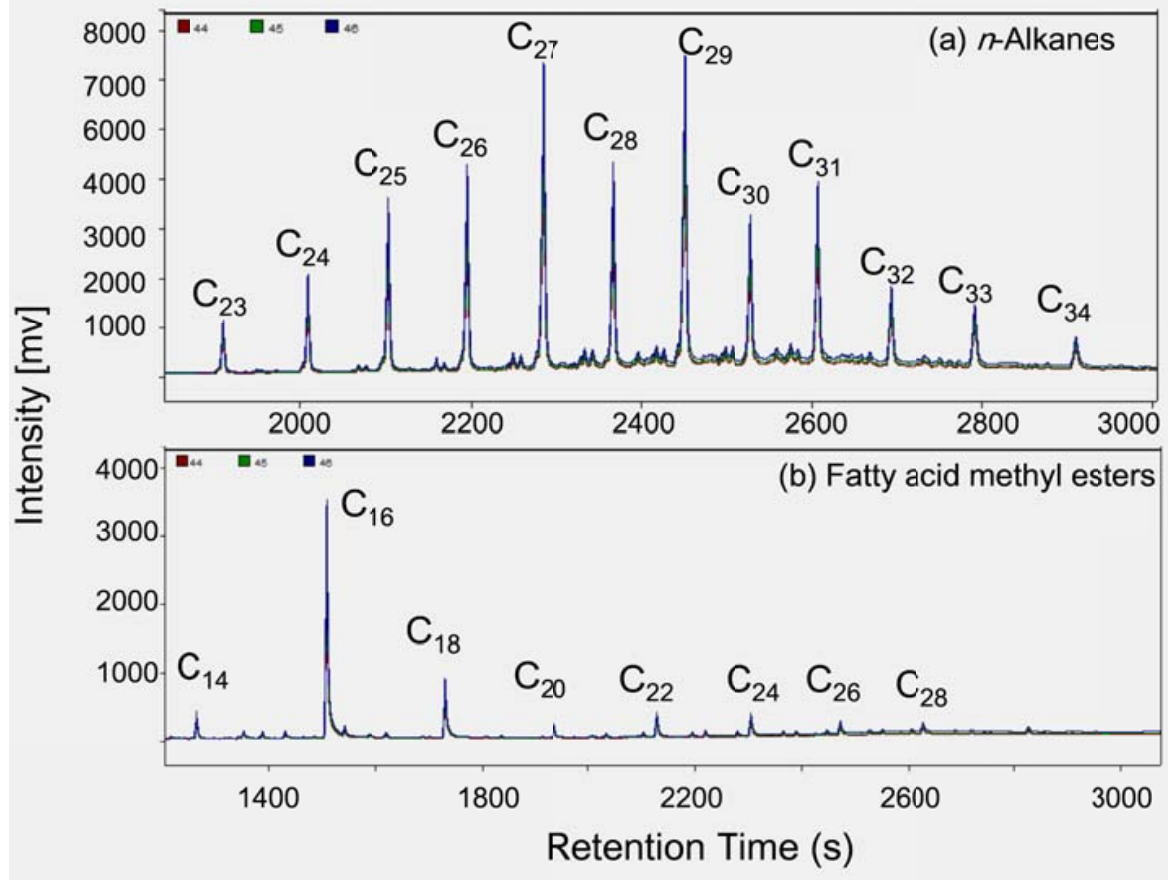

Figure S1. Typical isotope ratio gas chromatograms for total extracts of aerosol samples from Beijing. (a) n-Alkanes, (b) Fatty acid methyl esters. 


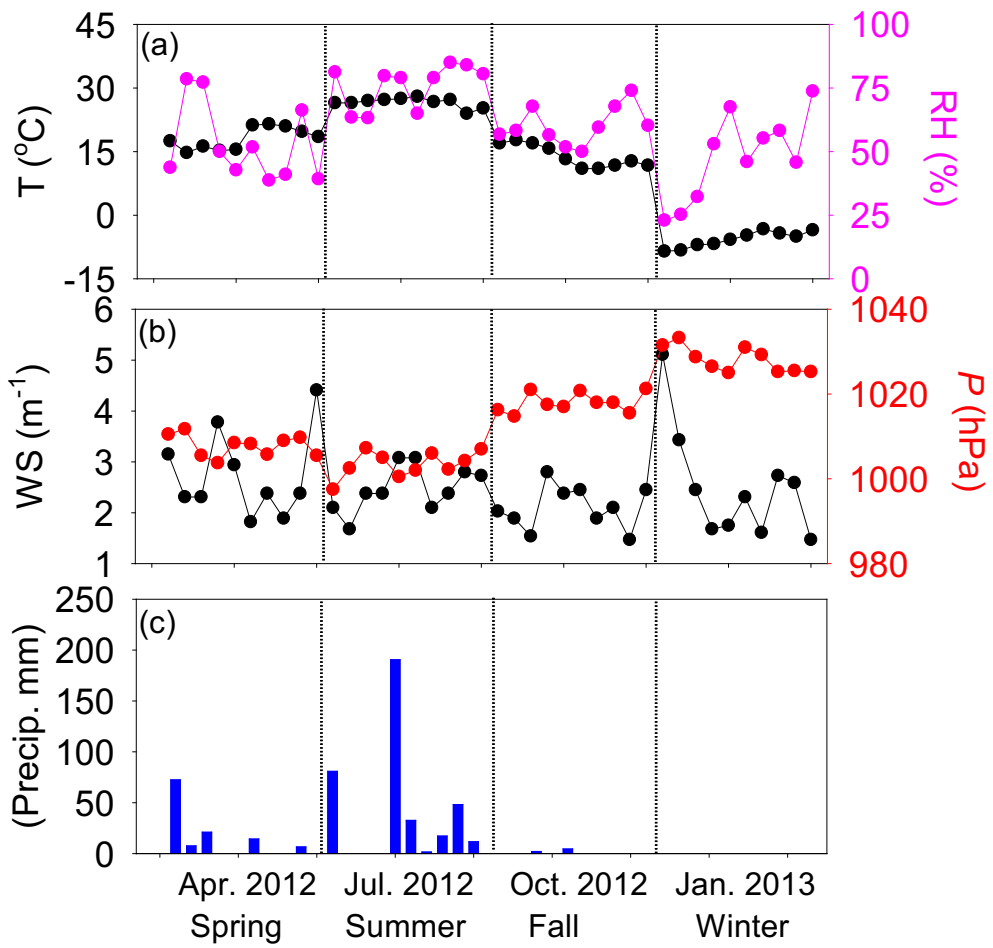

Figure S2. (a) Temperature and relative humidity (RH), (b) wind speed (WS) and pressure $(P),(\mathrm{c})$ precipitation (Precip.). The data were obtained from the automatic meteorological station at the sampling site ( $8 \mathrm{~m}$ above ground level). 


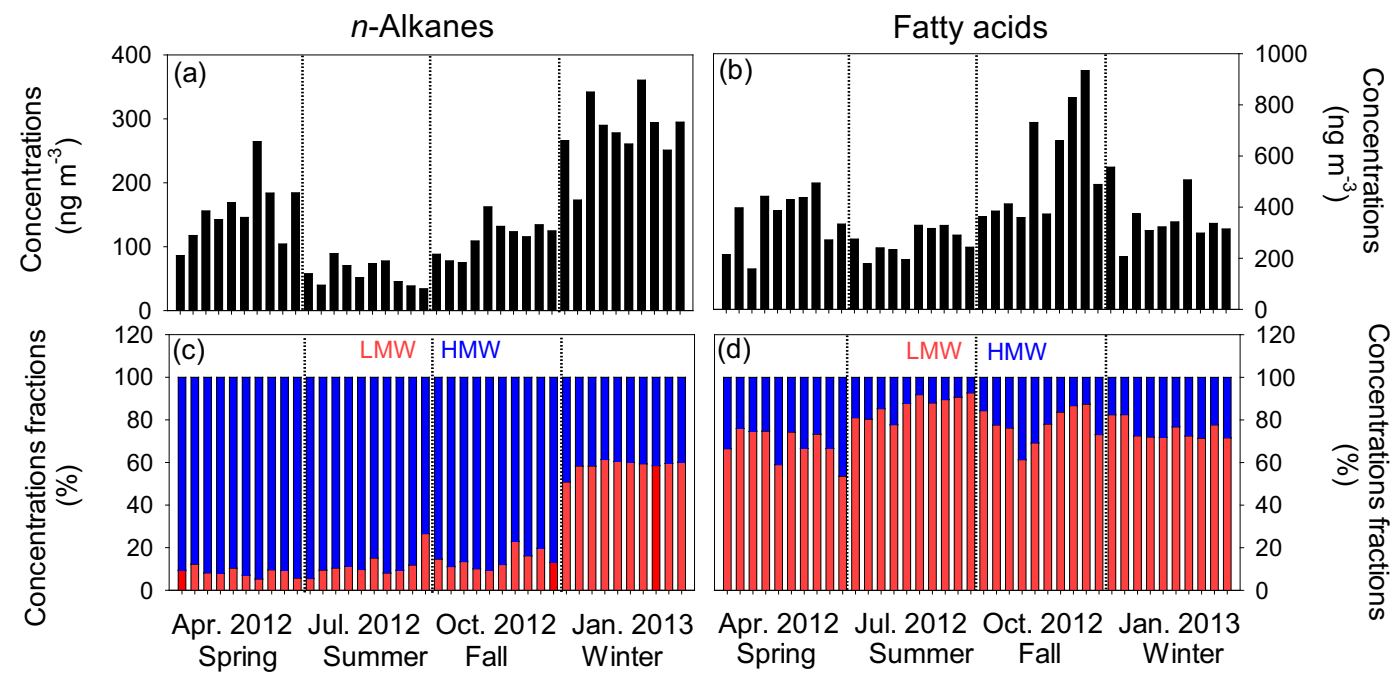

Figure S3. Temporal trends of concentrations of (a) $n$-alkanes and (b) fatty acids, and concentrations fractions of (c) $n$-alkanes and (d) fatty acids. 
(a)

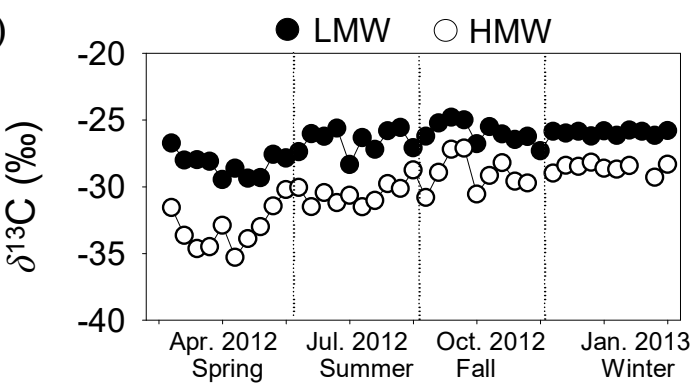

(b)
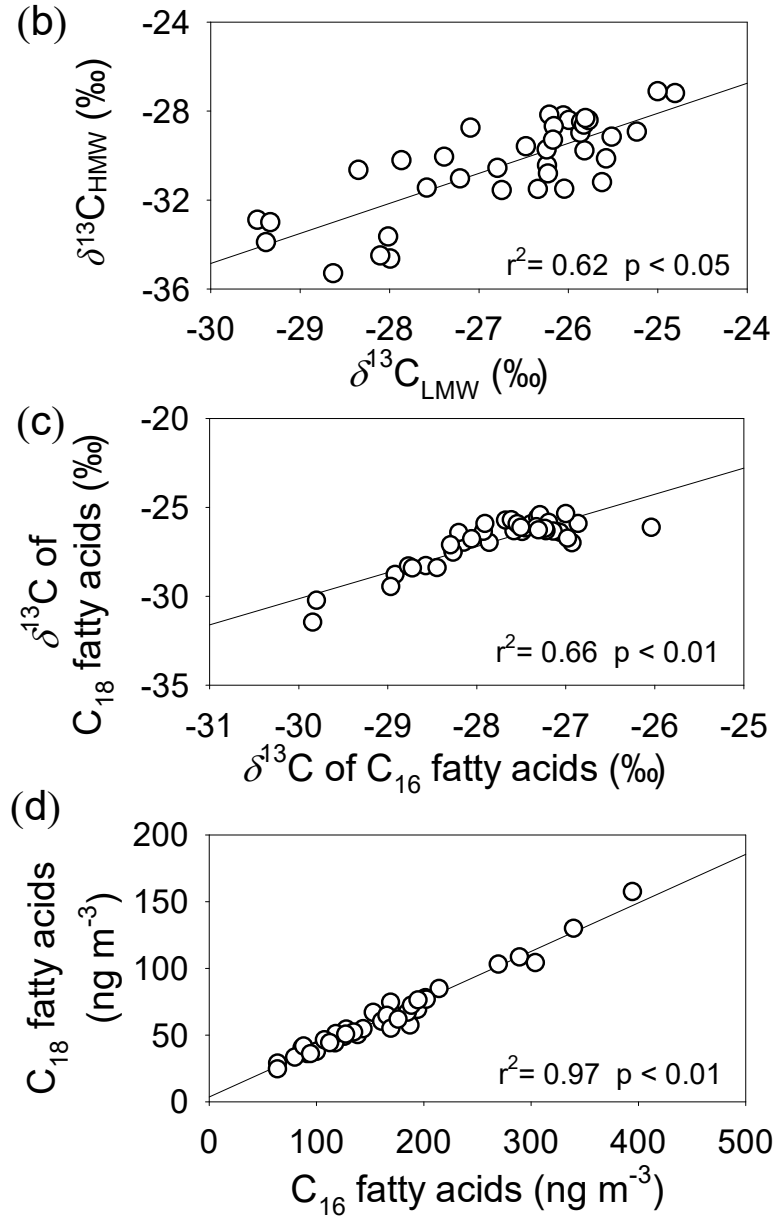

Figure S4. Time series of (a) $\delta^{13} \mathrm{C}$ values of HMW and LMW fatty acids, and (b) scatter plots of $\delta^{13} \mathrm{C}$ of LMW fatty acids versus $\delta^{13} \mathrm{C}$ of HMW fatty acids, and (c) scatter plots of $\delta^{13} \mathrm{C}$ of $\mathrm{C}_{16: 0}$ fatty acids versus $\delta^{13} \mathrm{C}$ of $\mathrm{C}_{18: 0}$ fatty acids, and (d) scatter plots of concentrations of $\mathrm{C}_{16: 0}$ fatty acid versus concentrations of $\mathrm{C}_{18: 0}$ fatty acids. 

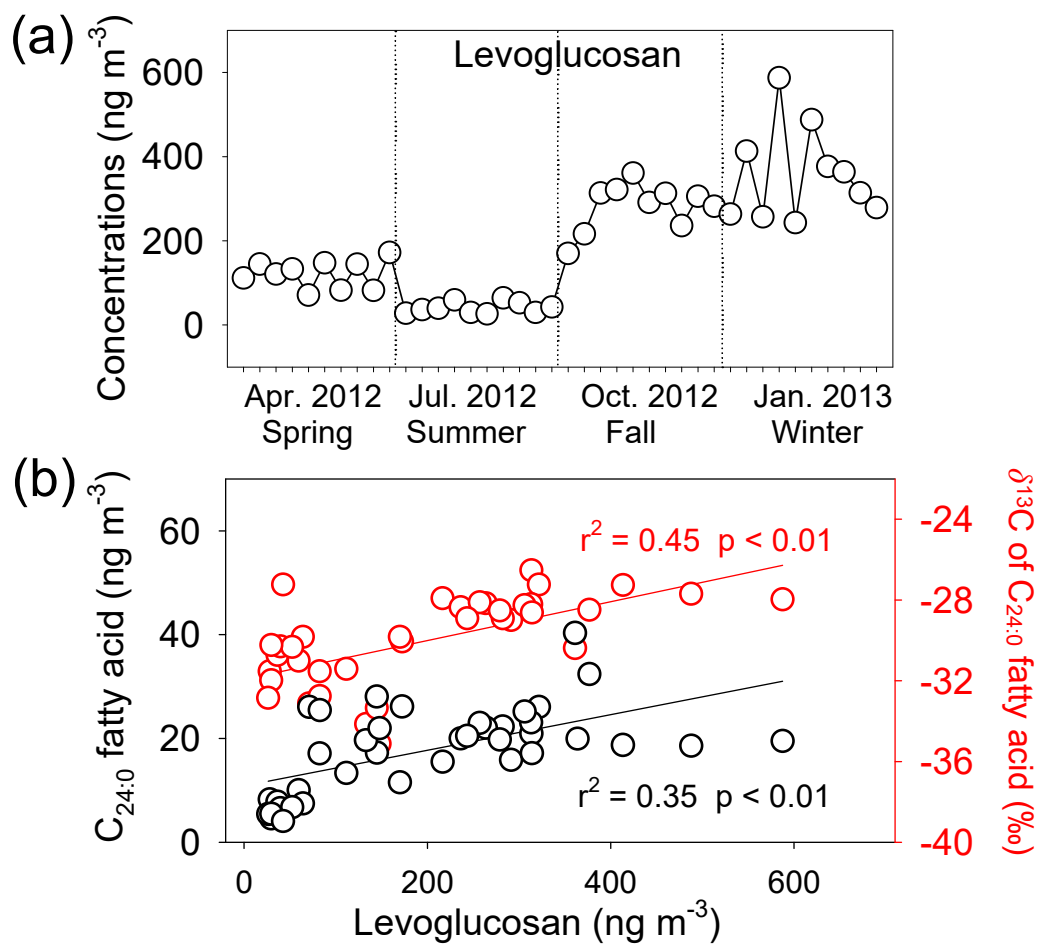

Figure S5. (a) Seasonal variations in the concentrations of levoglucosan, and (b)

Scatter plots of $\mathrm{C}_{24: 0}$ fatty acid versus concentrations of levoglucosan. 


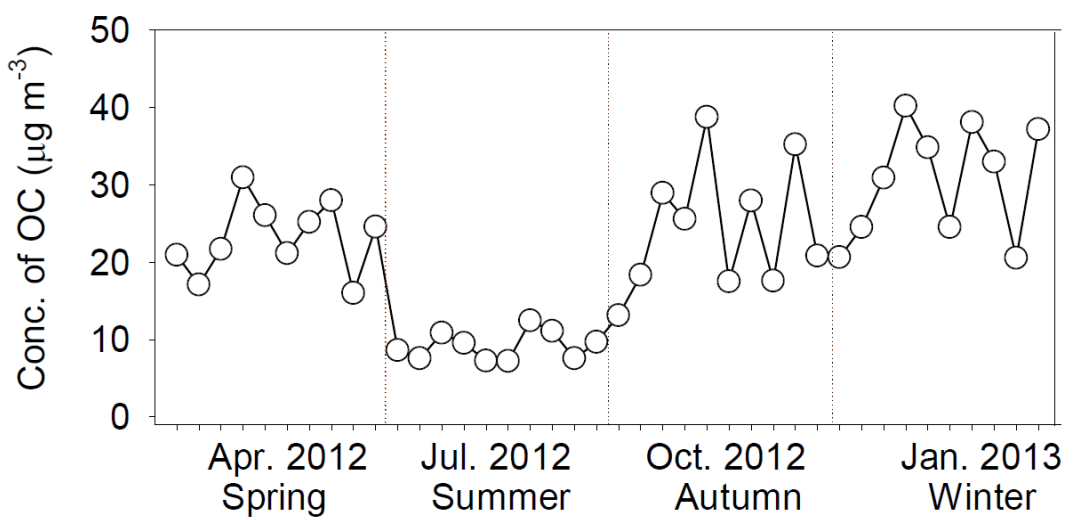

Figure S6. Temporal variations in aerosol OC in Beijing. 


\section{REFERENCES}

1. Ballentine, D. C.; Macko, S. A.; Turekian, V. C., Variability of stable carbon isotopic compositions in individual fatty acids from combustion of $\mathrm{C}_{4}$ and $\mathrm{C}_{3}$ plants: implications for biomass burning. Chem. Geol. 1998, 152, (1-2), 151-161.

2. Ballentine, D. C.; Macko, S. A.; Turekian, V. C.; Gilhooly, W. P.; Martincigh, B., Compound specific isotope analysis of fatty acids and polycycfic aromatic hydrocarbons in aerosols: impfications for biomass burning. Org. Geochem. 1996, 25, (1-2), 97-104.

3. Collister, J. W.; Rieley, G.; Stern, B.; Eglinton, G.; Fry, B., Compound-specific $\delta^{13} \mathrm{C}$ analyses of leaf lipids from plants with differing carbon dioxide metabolisms. Org. Geochem. 1994, 21, (6-7), $619-627$.

4. Bi, X. H.; Sheng, G. Y.; Liu, X. H.; Li, C.; Fu, J. M., Molecular and carbon and hydrogen isotopic composition of $n$-alkanes in plant leaf waxes. Org. Geochem. 2005, 36, (10), 1405-1417.

5. Chikaraishi, Y.; Naraoka, H., Compound-specific $\delta \mathrm{D}-\delta^{13} \mathrm{C}$ analyses of $n$-alkanes extracted from terrestrial and aquatic plants. Phytochemistry 2003, 63, (3), 361-371.

6. Harvey, S. D.; Jarman, K. H.; Moran, J. J.; Sorensen, C. M.; Wright, B. W., Characterization of diesel fuel by chemical separation combined with capillary gas chromatography (GC) isotope ratio mass spectrometry (IRMS). Talanta 2012, 99, (18), 262-269.

7. Bjoryøy, M.; Hall, K.; Gillyon, P.; Jumeau, J., Carbon isotope variations in $n$-alkanes and isoprenoids of whole oils. Chem. Geol. 1991, 93, (1), 13-20.

8. Muhammad, S. A.; Frew, R. D.; Hayman, A. R., Compound-specific isotope analysis of diesel fuels in a forensic investigation. Front. Chem. 2015, 3, 1-10.

9. Yamamoto, S.; Kawamura, K., Compound-specific stable carbon and hydrogen isotopic compositions of $n$-alkanes in urban atmospheric aerosols from Tokyo. Geochem.J. 2010, 44, (5), 419-430.

10. Fang, J. S.; Kawamura, K.; Ishimura, Y.; Matsumoto, K., Carbon isotopic composition of fatty acids in the marine aerosols from the Western North Pacific: Implication for the source and atmospheric transport. Environ. Sci. Technol. 2002, 36, (12), 2598-2604.

11. Matsumoto, K.; Kawamura, K.; Uchida, M.; Shibata, Y.; Yoneda, M., Compound specific radiocarbon and $\delta^{13} \mathrm{C}$ measurements of fatty acids in a continental aerosol sample. Geophys. Res. Lett. 2001, 28, (24), 4587-4590.

12. Bendle, J. A.; Kawamura, K.; Yamazaki, K., Seasonal changes in stable carbon isotopic composition of $n$-alkanes in the marine aerosols from the western North Pacific: Implications for the source and atmospheric transport. Geochim. Cosmochim. Acta 2006, 70, (1), 13-26. 